International Journal of Pure and Applied Mathematics

Volume 107 No. 4 2016, 927-937

ISSN: 1311-8080 (printed version); ISSN: 1314-3395 (on-line version)

url: http://www.ijpam.eu

doi: 10.12732 /ijpam.v107i4.10

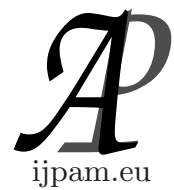

\title{
ON THE RELATIONSHIP BETWEEN
}

\section{SEVERAL CATEGORIES IN FUZZY UNIFORMITIES}

\author{
Maryam Yaghoubi \\ Department of Mathemematics \\ Payame Noor University \\ P.O. Box 19395-3697, IRAN
}

\begin{abstract}
Uniform structure on the $\mathbb{T}$-valued spaces are defined using different set of axioms and basic terms. A different approach to fuzzy uniformities, in terms of $\mathbb{T}$-covers was introduced which seems the most natural one.

The purpose of this paper is to present another characterization of fuzzy uniformities in the style of Weil that we call it $\mathbb{T}$ - Weil uniformity. We formulate and investigate a definition of entourage uniformity alternative to that one of Hutton. It is expressed in terms of coproduct of fuzzy spaces. We identify this new notion with the existing ones by proving the isomorphism between the respective categories.
\end{abstract}

AMS Subject Classification: 54A40, 06D20

Key Words: $\mathbb{T}$-Weil uniform spaces, $\mathbb{T}$-valued uniform spaces, Hutton uniform spaces

\section{Introduction}

Topological, proximal and uniform structures on the $\mathbb{T}$-valued spaces are defined using different set of axioms and basic terms. There are various equivalent definitions in literature even for each of these structures. Fuzzy uniformity was studied by three authors: B. Hutton in [4], R. Lowen in [6], U. Höhle in $[2,3]$. While all these authors, as a starting point, took certain counterpart of the filter approach to uniformities in fuzzy context (see [10], as different from the approach based on uniform covers, see [9], in set theory). The ideas on which

Received: January 15, 2016

Published: May 5, 2016 (c) 2016 Academic Publications, Ltd.

url: www.acadpubl.eu 
these authors based their works, the applied technique and the obtained results are essentially different.

In [7] the author present a different approach to fuzzy uniformities in terms of $\mathbb{T}$-covers that is called $\mathbb{T}$-valued uniformity.The purpose of this paper is to present another characterization of fuzzy uniformities in the style of Weil that we call it $\mathbb{T}$ - Weil uniformity. We formulate and investigate a definition of entourage uniformity alternative to that one of Hutton. It is expressed in terms of coproduct of fuzzy spaces. We identify this new notion with the existing ones by proving the isomorphism between the categories $\mathbb{T}$-Unif, $\mathbb{T}$-W Unif and Hutt-Unif.

Note. In this paper $\mathbb{T}$ will always denote a frame.

\section{Preliminaries}

Definition 2.1. (see [5]) A frame $\mathbb{T}$ is a complete lattice satisfying the distribution law $x \wedge \vee(A)=\vee\{x \wedge a \mid a \in A\}$, the bottom resp top of $\mathbb{T}$ will be denoted by 0 resp 1 .

Definition 2.2. (see [4]) Hutton generalized the concept of uniformity to fuzzy case as follows.

Let $\mathbb{T}$ be a complete lattice and let $H(\mathbb{T}, X)$ be the set of maps $e: \mathbb{T}^{X} \rightarrow \mathbb{T}^{X}$ which satisfies:

(1) $e(\phi)=\phi$,

(2) $e(\mu) \geq \mu$,

(3) $e\left(\vee_{i \in \Gamma} \mu_{i}\right)=\vee_{i \in \Gamma} e\left(\mu_{i}\right)$ for $\left\{\mu_{i}\right\}_{i \in \Gamma} \subset \mathbb{T}^{X}$.

For every $e, h \in H(\mathbb{T}, X)$, we have the following properties.

(1) For all $\mu \in \mathbb{T}^{X}, e^{-1}(\mu)=\wedge\left\{p \in \mathbb{T}^{X} \mid e\left(p^{\prime}\right) \leq \mu^{\prime}\right\}$. Then $e^{-1} \in H(\mathbb{T}, X)$.

(2) For all $\mu \in \mathbb{T}^{X}, e \wedge h(\mu)=\wedge\left\{e\left(\mu_{1}\right) \vee h\left(\mu_{2}\right) \mid \mu_{1} \vee \mu_{2}=\mu\right\}$ then $e \wedge h \in$ $H(\mathbb{T}, X)$.

(3) For all $\mu \in \mathbb{T}^{X}$,eoh $(\mu)=e(h(\mu))$. Then $e o h \in H(\mathbb{T}, X)$.

(4) $\alpha$ is $e$-small if $\alpha \leq e(\beta)$ whenever $\alpha \wedge \beta \neq 0$. 
A Hutton uniformity on $X$ is a subset $D$ of $H(\mathbb{T}, X)$ such that it satisfies the following axioms:

$$
\begin{array}{ll}
\left.H U_{1}\right) & D \neq \varnothing, \\
\left.H U_{2}\right) & e \in D \text { and } e \leq h \text { implies } h \in D, \\
\left.H U_{3}\right) & e \in D \text { and } h \in D \text { implies } e \wedge h \in D, \\
\left.H U_{4}\right) & e \in D \text { implies there exist } h \in D \text { such that } h o h \leq e, \\
\left.H U_{5}\right) & e \in D \text { implies } e^{-1} \in D .
\end{array}
$$

Definition 2.3. Let $(X, D),\left(X^{\prime}, D^{\prime}\right)$ be two Hutton uniform spaces $A$ mapping $f: X \rightarrow X^{\prime}$ is said to be uniformly homomorphism such that for every $e \in D$, there exists $g \in D^{\prime}$ with $g \cdot \vec{f} \leq \vec{f} \cdot e$. The resulting category will be denoted by Hutt-Unif.

Definition 2.4. (see [6]) Let $L$ be a frame. The coproduct of the frame $L$ by itself

$$
L \stackrel{u_{1}^{L}}{\rightarrow} L \oplus L \stackrel{u_{2}^{L}}{\rightarrow} L
$$

can be constructed as follows:

Take the Cartesian product $L \times L$ with the usual order $A$ down Set $A$ of $L \times L$ is a $C$ - ideal if $(\{x\} \times S \subseteq A \Rightarrow(x, \vee S) \in A)$ and $(S \times\{y\} \subseteq A \Rightarrow(\vee S, y) \in$ $A)$. Put $L \oplus L$ as the frame of all $C$-ideals of $L \times L$. Observe that the case $S=\phi$ implies that every $C$-ideal contains the set $D:=\downarrow\{(1,0)\} \cup \downarrow\{(0,1)\}$. Obviously, each $\downarrow\{(x, y)\} \cup \boldsymbol{O}$ is a $C$-ideal. It is denoted by $x \oplus y$. Finally put $u_{1}^{L}(x)=x \oplus 1$ and $u_{2}^{L}(y)=1 \oplus y$.

\section{3. $\mathbb{T}$-Valued Uniform Spaces and $\mathbb{T}$-Weil Uniform Spaces}

Definition 3.1. Let $\underline{1}$ denote the top element of $\mathbb{T}^{X}$, that is $\underline{1}: x \rightarrow 1$. We say that

$$
\mathcal{U}=\left(U_{i}: X \rightarrow \mathbb{T}\right)_{i \in I}
$$

is a $\mathbb{T}$-cover of $X$ if $\vee_{I} U_{i}=1$. The set $\mathbb{T}-\operatorname{Cov}(X)$ of all $\mathbb{T}$-covers of $X$ is preordered by $\mathcal{V} \leq \mathcal{U}$ (i.e. For each $V \in \mathcal{V}$ there exists $U \in \mathcal{U}$ such that $V \leq U)$.

Further let $\mathcal{U} \wedge \mathcal{V}:=\{U \wedge V \mid U \in \mathcal{U}, V \in \mathcal{V}\}$ 
For each $U \in \mathcal{U}$, let

$$
\operatorname{st}(U, \mathcal{U}):=\vee\{V \in \mathcal{U} \mid V \wedge U \neq 0\}
$$

and

$$
\mathcal{U}^{*}:=\{s t(U, \mathcal{U}) \mid U \in \mathcal{U}\} .
$$

Clearly $\mathcal{U} \leq \mathcal{U}^{*}$, since $U \leq \operatorname{st}(U, \mathcal{U})$ for every $U \in \mathcal{U}$. Therefore $\mathcal{U}^{*}$ is a $\mathbb{T}$-cover whenever $\mathcal{U}$ is a $\mathbb{T}$-cover.

We say that a pair $(X, \mu)$ consisting of a set $X$ and a non-empty family of $\mathbb{T}$-covers of $X$ is a covering $\mathbb{T}$-uniform space (see [8]) whenever the following conditions are satisfied:

(CU1) $\mathcal{U} \leq \mathcal{V}, \mathcal{U} \in \mu \Rightarrow \mathcal{V} \in \mu$.

(CU2) For every $\mathcal{U}, \mathcal{V} \in \mu, \mathcal{U} \wedge \mathcal{V} \in \mu$.

(CU3) For each $\mathcal{U} \in \mu$ there exists $\mathcal{V} \in \mu$ such that $\mathcal{V}^{*} \leq \mathcal{U}$.

A map $f:(X, \mu) \rightarrow(Y, v)$ is uniform homomorphism if, for every $\mathcal{V}:=$ $\left(V_{i}\right)_{I} \in v, f^{-1}[\mathcal{V}]:=\left(V_{i} \cdot f\right)_{I} \in \mu$. The resulting category will be denoted by $\mathbb{T}$-Unif. Of course, for $\mathbb{T}=2$, this is just the category of (covering) uniform spaces of Tukey, see [9].

Now let $(X, \mu) \in \mathbb{T}$ - Unif and define

$$
L_{\mu}:=\left\{U \in \mathbb{T}^{X} \mid U(x) \neq 0 \Rightarrow \exists \mathcal{U} \in \mu: \operatorname{st}(\hat{x}, \mathcal{U}) \leq U\right\},
$$

where $\hat{x}: X \rightarrow \mathbb{T}$ is defined by $\hat{x}(y)=1$ if $y=x$ and $\hat{x}(y)=0$ otherwise. Note that, when $\mathbb{T}=2, L_{\mu}$ is just the crisp topology $\mathcal{T}_{\mu}$ induced by the (classical) uniformity $\mu$ on $X$.

Proposition 3.2. $L_{\mu}$ is a subframe of $\mathbb{T}^{X}$.

Since $\mathbb{T}^{X}$ is a frame $\overline{0}$ and $\overline{1}$ denote the least and the greatest element in $\mathbb{T}^{X}$ we can define weil entourage on $L^{X}$.

Definition 3.3. (see [11]) $E \in \mathbb{T}^{X} \oplus \mathbb{T}^{X}$ is $\mathbb{T}$-Weil entourage of $\mathbb{T}^{X}$ if and only if $\left\{\mu \in \mathbb{T}^{X} \mid(\mu, \mu) \in E\right\}$ is a cover of $\mathbb{T}^{X}$. That is $\vee\left\{\mu \in \mathbb{T}^{X} \mid(\mu, \mu) \in E\right\}=\underline{1}$.

The collection $\mathbb{T}-W$ Ent $(X)$ of all $\mathbb{T}$-Weil entourage of $\mathbb{T}^{X}$ may be partially ordered by inclusion. 
Definition 3.4. We define the composition of $\mathbb{T}$-Weil entourage as follows:

$$
E o F=\vee\left\{f \oplus g \mid \exists h \in \mathbb{T}^{X} \backslash \underline{0},(f, h) \in E,(h, g) \in F\right\}
$$

The inverse of a $\mathbb{T}$ - Weil entourage $E$ has the natural definition $E^{-1}=\{(g, f) \mid(f$, $g) \in E\}$.

The element $\vee\left\{g \in \mathbb{T}^{X} \mid(g, g) \in E, f \wedge g \neq 0\right\}$ will be denoted by $\operatorname{st}(f, E)$.

We also consider a new partial order in $\mathbb{T}^{X}$, induced by a family $\mathcal{E}$ of $\mathbb{T}$ weil entourages:

$g \stackrel{\mathcal{E}}{\Delta} f(g$ is $\mathcal{E}$ - strongly below $f)$ if there is $E \in \mathcal{E}$ such that $E o(g \oplus g) \subseteq f \oplus f$.

When $\mathcal{E}$ is symmetric $\left(E \in \mathcal{E}\right.$ implies $E^{-1} \in \mathcal{E}$ ) this is equivalent to saying there is $E \in \mathcal{E}$ such that $(f \oplus f) o E \subseteq g \oplus g$.

Definition 3.5. Let $X$ be a nonempty set and $\mathcal{E} \subset \mathbb{T} w E n t(X)$. We say $(X, \mathcal{E})$ is a $\mathbb{T}$-Weil quasi uniformity on $X$ if it satisfies the following conditions:

$\left(\mathbb{T}-\mathrm{WQU}_{1}\right) \mathcal{E}$ is a filter of $(\mathbb{T}-W \operatorname{Ent}(X), \subseteq)$,

$\left(\mathbb{T}-\mathrm{WQU}_{2}\right)$ For each $E \in \mathcal{E}$ there is $F \in \mathcal{E}$ such that $F o F \subseteq E$.

The pair $(X, \mathcal{E})$ is said to be a $\mathbb{T}$-Weil quasi-uniform space.

A $\mathbb{T}$-Weil quasi uniform space $(X, \mathcal{E})$ is called a $\mathbb{T}$-Weil uniform space if it satisfies

$\left(\mathbb{T}-\mathrm{WU}_{3}\right)$ for any $E \in \mathcal{E}, E^{-1}$ is also in $\mathcal{E}$.

It is useful to note that the symmetric $\mathbb{T}$ - Weil entourages $E$ of $\mathcal{E}$ form a basis for $\mathcal{E}$. In fact, if $E \in \mathcal{E}$ then $E^{-1} \in \mathcal{E}$ so $E \cap E^{-1}$ is a symmetric $\mathbb{T}$-Weil entourage of $\mathcal{E}$ contained in $E$.

Definition 3.6. Let $(X, \mathcal{E}),\left(X^{\prime}, \mathcal{E}^{\prime}\right)$ be two $\mathbb{T}$-Weil uniform spaces. A mapping $f: X \rightarrow X^{\prime}$ is said to be uniformly homomorphic if $(\vec{f} \oplus \vec{f})(E) \in \mathcal{E}^{\prime}$ whenever $E \in \mathcal{E}$.

We will denote by $\mathbb{T}$-W Unif the category whose objects are $\mathbb{T}$-Weil uniform spaces and morphisms are uniformly homomorphism mappings. 


\section{The Isomorphism between the Categories $\mathbb{T}$-Unif, $\mathbb{T}-W$ Unif and Hutt-Unif}

The functor $\psi: \mathbb{T}$-Unif $\rightarrow \mathbb{T}-W$ Unif.

Let $\mu$ be a family of $\mathbb{T}-\operatorname{Cov}(X)$. For each $\mathcal{U} \in \mu$ consider the $\mathbb{T}$-Weil entourage $E_{\mathcal{U}}:=\bigvee_{U \in \mathcal{U}}(U \oplus U)$, and denote the set $\left\{E_{\mathcal{U}} \mid \mathcal{U} \in \mu\right\}$ by $\mathcal{E}_{\mu}$.

Proposition 4.1. Let $\mu$ be a $\mathbb{T}$-valued uniformity on a space $X$. Then $\mathcal{E}_{\mu}$ is a $\mathbb{T}$-Weil uniformity on $X$.

Proof. $\mathbb{T}$-WU1) Let $E_{\mathcal{U}}, E_{\mathcal{V}} \in \mathcal{E}_{\mu}$. Take $w \in \mu$ such that $w \leq \mathcal{U} \wedge \mathcal{V}$. Clearly $E_{w} \subseteq E_{\mathcal{U}} \cap E_{\mathcal{V}}$

$\mathbb{T}$-WU2) Let $E_{\mathcal{U}} \in \mathcal{E}_{\mu}$, and take $\mathcal{V} \in \mu$ such that $\mathcal{V}^{*} \leq \mathcal{U}$. Then $E_{\mathcal{V} o E_{\mathcal{V}} \subseteq}$ $E_{\mathcal{U}}$ : By Lemma 4.4.3(a) it follows that

$$
E_{\mathcal{V}} o E_{\mathcal{V}}=\left(\bigcup_{U \in \mathcal{V}}(U \oplus U)\right) o\left(\bigcup_{U \in \mathcal{V}}(U \oplus U)\right)
$$

Let $(f, g) \in E_{\mathcal{V}} \circ E_{\mathcal{V}}$ then $(f, h) \leq(U, U)$, and $(h, g) \leq(V, V)$ where $U, V \in \mathcal{V}$ and $h \neq \underline{0}$. Then $V \wedge U \neq \underline{0}, f \leq U \leq \operatorname{st}(U, \mathcal{V})$ and $g \leq V \leq s t(U, \mathcal{V})$. As $\operatorname{st}(U, \mathcal{V}) \in \mathcal{V}^{*} \leq \mathcal{U}$, this says that there is $W \in \mathcal{U}$ such that $f \leq W, g \leq W$ and consequently, that $(f, g) \in E_{\mathcal{U}}$.

$\mathbb{T}$-WU3) the symmetry condition is obviously satisfied since each $E_{\mathcal{U}}$ is symmetric.

In the sequel, for every uniformity $\mu, \psi(\mu)$ denotes the $\mathbb{T}$-Weil uniformity $\mathcal{E}_{\mu}$. The correspondence $(X, \mu) \rightarrow(X, \psi(\mu))$ is functorial. Indeed, it is the function on objects of a functor $\psi: \mathbb{T}$ - Unif $\rightarrow \mathbb{T}-W$ Unif whose function on morphisms is described in the following proposition

Proposition 4.2. Let $(X, \mu)$ and $\left(X^{\prime}, \mu^{\prime}\right)$ be covering $\mathbb{T}$-uniform spaces and let $f:(X, \mu) \rightarrow\left(X^{\prime}, \mu^{\prime}\right)$ be a uniform homomorphism. Then $f:(X, \psi(\mu)) \rightarrow$ $\left(X^{\prime}, \psi\left(\mu^{\prime}\right)\right)$ is a $\mathbb{T}$ - Weil uniform homomorphism.

Proof. It is obvious since, for every $\mathcal{U} \in \mu$,

$$
\left(f^{\rightarrow} \oplus f^{\rightarrow}\right)\left(E_{\mathcal{U}}\right)=\bigvee_{U \in \mathcal{U}}\left(f^{\rightarrow}(U) \oplus f^{\rightarrow}(U)\right)=E_{f \rightarrow[\mathcal{U}]}
$$


The functor $\Phi: \mathbb{T}-W$ Unif $\rightarrow$ Hutt-Unif.

Let $\mathcal{E} \subseteq \mathbb{T}^{X} \oplus \mathbb{T}^{X}$. For each $E \in \mathcal{E}$ define $e_{E}: \mathbb{T}^{X} \rightarrow \mathbb{T}^{X}$ by $e_{E}(U)=$ $\operatorname{st}(U, E)$ and denote the set $\left\{e_{E} \mid E \in \mathcal{E}\right\}$ by $\mathbf{D}_{\mathcal{E}}$. It is obvious that each $e_{E}$ preserves arbitrary joins and that it is an entourage of $\mathbb{T}^{X}$ whenever $E$ is a $\mathbb{T}$-Weil entourage.

Proposition 4.3. Let $\mathcal{E}$ be a $\mathbb{T}$-Weil uniformity on a space $X$. Then $\boldsymbol{D}_{\mathcal{E}}$ is a Hutton uniformity on $X$.

Proof. $\mathbf{D}_{\mathcal{E}}$ satisfies the axiom (1)-(3).

(1) for all $E \in \mathcal{E}, e_{E}(\varnothing)=s t(\varnothing, E)=\varnothing$

(2) for all $E \in \mathcal{E}, e_{E}(U)=s t(U, E) \geq U$

(3) for all $E \in \mathcal{E} e_{E}\left(\vee_{i} U_{i}\right)=s t\left(\vee_{i} U_{i}, E\right)=\vee_{i} s t\left(U_{i}, E\right)$. Then $D_{\mathcal{E}} \subset \Omega\left(\mathbb{T}^{X}\right)$.

HU1) $\mathbf{D}_{\mathcal{E}} \neq \phi$ since every C-ideal contains the C-ideal $\Phi$ then there exist $\varnothing \neq E \in \mathcal{E}$ such that $e_{E} \in \mathbf{D}_{\mathcal{E}}$.

HU2, HU3) $e_{E}, e_{F} \in \mathbf{D}_{\mathcal{E}}$ then $E, F \in \mathcal{E}$ in order to prove that $\mathbf{D}_{\mathcal{E}}$ is a filter basis just take for some $\mathbb{T}$-Weil entourage $G$ such that $G \subseteq E \cap F$.

HU4) For $e_{E} \in \mathbf{D}_{\mathcal{E}}$ consider $F \in \mathcal{E}$ such that $F^{2} \subset E$ by 4.4.7(d). We observed that $\operatorname{st}(s t(U, F), F) \leq s t\left(U, F^{2}\right)$. Hence $e_{F}^{2} \leq e_{F^{2}} \leq e_{E}$.

HU5) $e_{E} \in \mathbf{D}_{\mathcal{E}}$ we have that

$$
\begin{aligned}
& V \wedge e_{E}(U)=V \wedge s t(U, E)=\underline{0} \Leftrightarrow \vee\{V \wedge W \mid(W, W) \in E, W \wedge U \neq \underline{0}\}=\underline{0}, \\
& e_{E}(V) \wedge U=\operatorname{st}(V, E) \wedge U=\underline{0} \Leftrightarrow \vee\{W \wedge U \mid(W, W) \in E, W \wedge V \neq \underline{0}\}=\underline{0} .
\end{aligned}
$$

These two formula are equivalent, then we have,

$$
\begin{aligned}
e_{E}^{-1}(U) & =\wedge\left\{V \mid e_{E}\left(V^{\prime}\right) \leq U^{\prime}\right\}=\wedge\left\{V \mid e_{E}\left(V^{\prime}\right) \wedge U \leq \underline{0}\right\} \\
& =\wedge\left\{V \mid V^{\prime} \wedge e_{E}(U) \leq \underline{0}\right\}=\wedge\left\{V \mid e_{E}(U) \leq V\right\}=e_{E}(U) .
\end{aligned}
$$

In what follows, if $\mathcal{E}$ is a $\mathbb{T}$-Weil uniformity on $X$, then $\phi(\mathcal{E})$ denotes the Hutton uniformity. The correspondence $(X, \mathcal{E}) \rightarrow(X, \phi(\mathcal{E}))$ is functorial.

Proposition 4.4. Let $(X, \mathcal{E})$ and $\left(X^{\prime}, \mathcal{E}^{\prime}\right)$ be $\mathbb{T}$-Weil uniform spaces and let $f:(X, \mathcal{E}) \rightarrow\left(X^{\prime}, \mathcal{E}^{\prime}\right)$ be a $\mathbb{T}$-Weil uniform homomorphism. Then $f$ : $(X, \phi(\mathcal{E})) \rightarrow\left(X^{\prime}, \phi\left(\mathcal{E}^{\prime}\right)\right)$ is a Hutton uniform homomorphism. 
Proof. Let $e_{E} \in \mathbf{D}_{\mathcal{E}}$ where $E \in \mathcal{E}$. Take a symmetric $F \in \mathcal{E}$ such that $F^{2} \subseteq E$. Since $f$ is a Weil uniform homomorphism, $(f \rightarrow \oplus f \rightarrow)(F) \in \mathcal{E}^{\prime}$. In order to show that $f:(X, \phi(\mathcal{E})) \rightarrow\left(X^{\prime}, \phi\left(\mathcal{E}^{\prime}\right)\right)$ is uniform it suffices to show that

$$
e_{(f \rightarrow \oplus f \rightarrow)(F)} \cdot f \rightarrow \leq f \rightarrow \cdot e_{E} .
$$

So, fix $\alpha \in \mathbb{T}^{X}$ and take $\beta \in \mathbb{T}^{X^{\prime}}$ such that $(\beta, \beta) \in\left(f \rightarrow \oplus f^{\rightarrow}\right)(F)$ and $(\beta \wedge f \rightarrow(\alpha)) \neq \underline{0}, \beta<e_{(f \rightarrow \oplus f \rightarrow)(F)}^{f \rightarrow(\alpha)}$. Then $(\beta, \beta \wedge f \rightarrow(\alpha)) \in\left(f \rightarrow \oplus f^{\rightarrow}\right)(F)$ and $(\beta \wedge f \rightarrow(\alpha), f \rightarrow(\alpha)) \in f^{\rightarrow}(\alpha) \oplus f^{\rightarrow}(\alpha)$ and consequently $(\beta, f \rightarrow(\alpha)) \in$ $\left(\left(f^{\rightarrow} \oplus f^{\rightarrow}\right)(F) o\left(f \rightarrow(\alpha) \oplus f^{\rightarrow}(\alpha)\right)\right.$. Further, since $F$ is of the form $\vee_{\gamma \in \Gamma}\left(a_{\gamma} \oplus b_{\gamma}\right)$ for some subset $\left\{\left(a_{\gamma}, b_{\gamma}\right) \mid \gamma \in \Gamma\right\}$ of $\mathbb{T}^{X} \times \mathbb{T}^{X}$,

$$
\begin{aligned}
& \left(f \rightarrow \oplus f^{\rightarrow}\right)(F) o\left(f^{\rightarrow}(\alpha) \oplus f^{\rightarrow}(\alpha)\right)=\left(f^{\rightarrow} \oplus f^{\rightarrow}\right)\left(\vee\left(a_{\gamma} \oplus b_{\gamma}\right)\right) o f \rightarrow(\alpha) \oplus f^{\rightarrow}(\alpha) \\
& =k\left(\bigcup_{\gamma \in \Gamma} f^{\rightarrow}\left(a_{\gamma}\right) \oplus f^{\rightarrow}\left(b_{\gamma}\right)\right) o k\left(\downarrow\left(f^{\rightarrow}(\alpha), f^{\rightarrow}(\alpha)\right)\right. \\
& =\cup_{\gamma \in \Gamma}\left(f ^ { \rightarrow } ( a _ { \gamma } ) \oplus f ^ { \rightarrow } ( b _ { \gamma } ) o \left(\downarrow\left(f^{\rightarrow}(\alpha), f^{\rightarrow}(\alpha)\right)\right.\right. \\
& \subset\left(f \rightarrow e_{E}\right)(\alpha) \oplus f^{\rightarrow}(\alpha) \text {. }
\end{aligned}
$$

For any $(a, b) \in\left(\cup_{\gamma \in \Gamma} f^{\rightarrow}\left(a_{\gamma}\right) \oplus f^{\rightarrow}\left(b_{\gamma}\right)\right) o \downarrow\left(f^{\rightarrow}(\alpha), f^{\rightarrow}(\alpha)\right) \backslash \emptyset \quad \exists c \in L^{X} \backslash \underline{0}$ and $\gamma \in \Gamma \quad(a, c) \leq\left(f \rightarrow\left(a_{\gamma}\right), f^{\rightarrow}\left(b_{\gamma}\right)\right)$ and $(c, b) \leq\left(f \rightarrow(\alpha), f^{\rightarrow}(\alpha)\right)$ it follows that $a \leq f \rightarrow\left(a_{\gamma} \vee b_{\gamma}\right)$ and therefore $a \leq\left(f \rightarrow \cdot e_{E}\right)(\alpha)$ indeed $\left(a_{\gamma} \vee b_{\gamma}\right) \wedge \alpha \neq \underline{0}$ because $f \rightarrow\left(b_{\gamma} \wedge \alpha_{\gamma}\right) \geq c \neq \underline{0}$ and by the symmetry of $F \quad\left(a_{\gamma} \vee b_{\gamma}, a_{\gamma} \vee b_{\gamma}\right) \in F^{2} \subseteq$ $E$ inclusion $a \leq f \rightarrow\left(a_{\gamma} \vee b_{\gamma}\right)<f \rightarrow\left(e_{E}(\alpha)\right)$ then $(a, b) \in\left(f \cdot e_{E}\right)(\alpha) \oplus f(\alpha)$. We have that $(\beta, f(\alpha)) \in\left(f^{\rightarrow} \oplus f^{\rightarrow}\right)(F) o\left(f \rightarrow(\alpha) \oplus f^{\rightarrow}(\alpha)\right) \subseteq\left(f^{\rightarrow} \cdot e_{E}\right)(\alpha) \oplus f^{\rightarrow}(\alpha)$. Hence $\beta \leq\left(f \rightarrow \cdot e_{E}\right)(\alpha)$ which implies that $e_{(f \rightarrow \oplus f \rightarrow)(F)} f^{\rightarrow}(\alpha) \leq f^{\rightarrow}\left(e_{E}\right)(\alpha)$ then $e_{(f \rightarrow \oplus f \rightarrow(F)} f \rightarrow \leq f \rightarrow \cdot e_{E}$.

We shall denote the functor defined above by $\phi$.

The functor $\theta$ : Hutt-Unif $\rightarrow \mathbb{T}$ - Unif:

Finally, we want to study that the functors $\theta$ between the categories Hutton uniform space and $\mathbb{T}$-valued uniform space. For each entourage $e$ of $\mathbb{T}^{X}$ let $\mathcal{U}_{e}$ be the cover of all $e$-small elements of $\mathbb{T}^{X}$ i.e. $\mathcal{U}_{e}=\left\{U \in \mathbb{T}^{X} \mid U \leq e(V), U \wedge V \neq \underline{0}\right\}$.

Proposition 4.5. Let $\boldsymbol{D}$ be Hutton uniformity on $X$, then $\mu_{\boldsymbol{D}}=\left\{\mathcal{U}_{e} \mid e \in\right.$ $D\}$ is a $\mathbb{T}$-valued uniformity on $X$.

Proof. $\left.C U_{1}, C U_{2}\right)$ Consider $\mathcal{U}_{e}, \mathcal{U}_{f} \in \mu_{\mathbf{D}}$ and let $g \in \mathbf{D}$ such that $g \leq e \wedge f$ then it is obvious that $\mathcal{U}_{g} \leq \mathcal{U}_{e} \wedge \mathcal{U}_{f}$. 
$\left.C U_{3}\right)$ Let $\mathcal{U}_{e} \in \mu_{\mathbf{D}}$ and take $f \in \mathbf{D}$ such that $f^{3} \leq e$ we claim $\mathcal{U}_{f}^{*} \leq \mathcal{U}_{e}$ consider $\operatorname{st}\left(U, \mathcal{U}_{f}\right) \in \mathcal{U}_{f}^{*}$. It suffices to show that $\operatorname{st}\left(U, \mathcal{U}_{f}\right)$ is $e$-small. So, consider $V \in \mathbb{T}^{X}$ such that $V \wedge s t\left(U, \mathcal{U}_{f}\right) \neq 0$ then there is $W \in \mathcal{U}_{f}$ with $W \wedge U \neq 0$ and $V \wedge W \neq 0$ then $f$-smallness of $U, W$ then $U \leq f(W) \leq f^{2}(V)$. Therefore for every $W^{\prime} \in \mathcal{U}_{f}$ such that $W^{\prime} \wedge U \neq \underline{0}$ we have $W^{\prime} \leq f(U) \leq$ $f^{3}(V) \leq e(V)$.Then $s t\left(U, \mathcal{U}_{f}\right)$ is e-small.

In the sequel, if $\mathbf{D}$ is a Hutton uniformity, $\theta(\mathbf{D})$ denotes the uniformity generated by $\mathcal{U}_{\mathbf{D}}$.

Proposition 4.6. Let $(X, \boldsymbol{D}) \rightarrow\left(X^{\prime}, \boldsymbol{D}^{\prime}\right)$ be a Hutton uniform homomorphism then $f:(X, \theta(\boldsymbol{D})) \rightarrow\left(X^{\prime}, \theta\left(\boldsymbol{D}^{\prime}\right)\right)$ is a covering $\mathbb{T}$-uniform homomorphism.

Proof. Let $\mathcal{U} \in \theta(\mathbf{D})$ and $e \in \mathbf{D}$ such that $\mathcal{U}_{e^{3}} \leq \mathcal{U}$ there exists $g \in \mathbf{D}^{\prime}$ with $g \cdot \vec{f} \leq \vec{f} \cdot e$. We show that $\mathcal{U}_{g} \leq \vec{f}\left[\mathcal{U}_{e^{3}}\right]$. Let $U$ be a non-zero $g$-small element of $\mathbb{T}^{X}$. Since $\vec{f}\left[\mathcal{U}_{e}\right]$ is a cover of $\mathbb{T}^{X^{\prime}}$, there exists $V \in \mathcal{U}_{e}$ satisfying $U \wedge \vec{f}(V) \neq 0$. Consequently $U \leq g \cdot \vec{f}(V) \leq \vec{f} \cdot e(V)$. But as can be easily proved, the fact $V$ is $e$-small implies that $e(V)$ is $e^{3}$-small. In conclusion $\mathcal{U}_{g} \leq \vec{f}\left[\mathcal{U}_{e^{3}}\right] \leq \vec{f}(\mathcal{U})$ and $\vec{f}(\mathcal{U}) \in \theta\left(D^{\prime}\right)$.

The Isomorphism. Finally, let us show that the functors $\psi, \Phi$ and $\Theta$ define an isomorphism between the categories $\mathbb{T}$-Unif, $\mathbb{T}$-W Unif and Hutt-Unif.

Lemma 4.7. For any $\mathbb{T}$-cover $\mathcal{U}$ of $X$ we have that:

(a) $\mathcal{U} \leq \mathcal{U}_{e_{E_{\mathcal{U}}}}$,

(b) $\mathcal{U}_{e_{E_{\mathcal{U}}}} \leq \mathcal{U}^{*}$

Proof. (a) Let $U \in \mathcal{U}$. For any $V \in \mathbb{T}^{X}$ satisfying $U \wedge V \neq \underline{0}$, since $(U, U) \in E_{\mathcal{U}}, U \leq s t\left(V, E_{\mathcal{U}}\right)=e_{E_{\mathcal{U}}}(V)$, that is, $U$ is $e_{E_{\mathcal{U}}}$ - small and therefore $U \in \mathcal{U}_{e_{E_{\mathcal{U}}}}$.

(b) for any non-zero $e_{E_{\mathcal{U}}}$-small member $U$ of $\mathbb{T}^{X}$, there exists $V \in \mathcal{U}$ such that $U \wedge V \neq \underline{0}$.

Then $U \leq \operatorname{st}\left(V, E_{\mathcal{U}}\right)$. But, for every $(W, W) \in E_{\mathcal{U}}, W$ is $\mathcal{U}$-small so $W \leq$ $\operatorname{st}(V, \mathcal{U})$ in case $W \wedge V \neq 0$. This means that $\operatorname{st}\left(V, E_{\mathcal{U}}\right) \leq \operatorname{st}(V, \mathcal{U})$. Hence $U \leq \operatorname{st}(V, \mathcal{U}) \in \mathcal{U}^{*}$. 
Lemma 4.8. For any symmetric $\mathbb{T}$-Weil entourage $E$ of $X$ we have:

(a) $E \subseteq E_{\mathcal{U}_{E^{2}}}$,

(b) $E_{\mathcal{U}_{E}} \subseteq E^{2}$.

Proof. (a) Consider $(U, V) \in E \backslash D$ because $(V, U) \in E \backslash \emptyset$ then $(U, U),(V, V)$, $(U \vee V, U \vee V)$ belong to $E^{2}$. Since every member of $E^{2}$ with equal coordinates

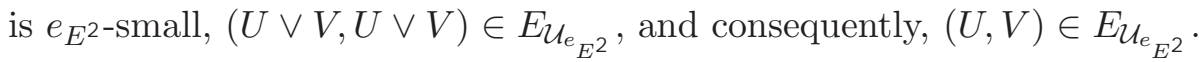

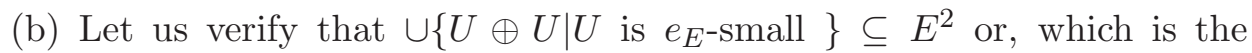
same, that $(U, U) \in E^{2}$ whenever $U$ is $e_{E}$-small consider $U \neq \underline{0}, e_{E}$-small. We have that $U \leq \vee\left\{W \in \mathbb{T}^{X} \mid(W, W) \in E, U \wedge W \neq \underline{0}\right\}$. Since $V$ is $e_{E^{-}}$ small, we get $U \leq e(W)=\vee\left\{V \in \mathbb{T}^{X} \mid(V, V) \in E, V \wedge W \neq \underline{0}\right\}$, for any $W$ such that $(W, W) \in E$ and $U \wedge W \neq \underline{0}$. For each $V$ in this set we have that $(W, W \wedge V),(W \wedge V, V) \in E$, which implies that $(W, V) \in E^{2}$. Therefore $(W, U) \in E^{2}$ and, consequently $(U, U) \in E^{2}$.

Lemma 4.9. Let $e$ be a symmetric entourage of $\mathbb{T}^{X}$. Then:

(a) $e \leq e_{E_{\mathcal{U}^{3}}}$,

(b) $e_{E_{\mathcal{U}}} \leq e$.

Proof. For any $U \in \mathbb{T}^{X}, e_{E_{\mathcal{U}^{3}}}(U)=s t\left(U, E_{\mathcal{U}_{e^{3}}}\right)$. On the other hand, $e(U)=$ $e(\vee\{U \wedge V \mid V$ is $e$-small $\})=\vee\{e(U \wedge V) \mid V$ is $e$ small and $U \wedge V \neq \underline{0}\}$. Consider any $e$-small element $V$ such that $U \wedge V \neq \underline{0}$. We have $e(U \wedge V)$ is $e^{3}$-small so $(e(U \wedge V), e(U \wedge V)) \in E_{\mathcal{U}_{e^{3}}}$. Since $e(U \wedge V) \wedge(U \wedge V)=U \wedge V \neq \underline{0}$. It follows that

$$
e(U \wedge V) \leq e_{E_{\mathcal{U}^{3}}}(U \wedge V) \leq e_{E_{\mathcal{U}^{3}}}(U) .
$$

Hence $e(U) \leq e_{E_{\mathcal{U}^{3}}}(U)$.

(b) $V$ is $\mathcal{U}_{e}$ - small whenever $(V, V) \in E_{\mathcal{U}_{e}}$. Therefore, $e_{E_{\mathcal{U}_{e}}}=\vee\{V \in$ $\left.\mathbb{T}^{X} \mid(V, V) \in E_{\mathcal{U}_{e}}, V \wedge U \neq \underline{0}\right\} \leq \operatorname{st}\left(U, \mathcal{U}_{e}\right) \leq e(U)$.

Proposition 4.10. Let $\mu, \mathcal{E}$ and $\boldsymbol{D}$ denote, respectively, a covering $\mathbb{T}$ uniformity and $\mathbb{T}$-Weil uniformity and Hutton - uniformity on $X$. Then

$$
\Theta \phi \psi(\mu)=\mu, \psi \Theta \phi(\mathcal{E})=\mathcal{E}
$$

and

$$
\phi \psi \Theta(\boldsymbol{D})=\boldsymbol{D} .
$$


Proof. The uniformity $\theta \phi \psi(\mu)$ has $\left\{\mathcal{U}_{e} \mid e \in \phi \psi(\mu)\right\}$ as a basis. It suffices to prove this is a basis for $\mu$, by Lemma 4.5.7 (a) $\left\{\mathcal{U}_{e} \mid e \in \phi \psi(\mathcal{U})\right\} \subseteq \mu$, and, by (b), for any $\mathcal{U} \in \mu$ there is some $\mathcal{V} \in \mu$ such that $\mathcal{U}_{e_{E_{\mathcal{V}}}} \subseteq \mathcal{U}$.

By Lemma 4.8 implies that the basis $\left\{E_{\mathcal{U}} \mid \mathcal{U} \in \theta \phi(\mathcal{E})\right\}$ of $\psi \theta \phi(\mathcal{E})$ is also a basis for $\mathcal{E}$, which proves the second equality, and Lemma 4.9 implies that the basis $\left\{e_{E} \mid E \in \psi \theta(\mathbf{D})\right\}$ of $\phi \psi \theta(\mathbf{D})$ is also a basis for $D$, which proves the equality $\phi \psi \theta(\mathbf{D})=\mathbf{D}$.

Theorem 4.11. The categories $\mathbb{T}$-Unif, $\mathbb{T}$ - $W$ Unif and Hutt -Unif are isomorphic.

Proof. The proof follows from Proposition 4.10.

\section{References}

[1] C.L. Chang, Fuzzy topological spaces, J. Math. Anal. Appl., 24 (1968), 182-190.

[2] U. Höhle, Probabilistic uniformization of fuzzy uniformities, Fuzzy Sets and Systems, 2 (1978), 311-332.

[3] U. Höhle, Probabilistic topologies induced by L-fuzzy uniformities, Manuscripta Math., 38 (1982), 289-323.

[4] B. Hutton, Uniformities on fuzzy topological spaces, J. Math. Anal. Appl., 58 (1977), 559-571.

[5] P.T. Johnstone, Stone Spaces, Cambridge Studies in Advanced Mathematics, 3, Cambridge University Press (1982).

[6] R. Lowen, Fuzzy uniform spaces, J. Math. Anal. Appl., 82 (1981), 370-385.

[7] J. Gutiérrez Garcia, U. Hohle, M.A. de Prada Vicente, On lattice-valued frames: The completely distributive case, Fuzzy Sets and Systems, 161 (2010), 1022-1030.

[8] A. Pultr, Pointless uniformities I. Complete regularity, Comment. Math. Unive. Carolin, 25, (1984), 91-104.

[9] J.W. Tukey, Convergence and uniformity in topology, Ann. Math. Studies, No. 1, Princeton University Press (1940).

[10] A. Weil, sur les Spaces ã structure uniformer et sur la topologies général, Act. Sci. et. Ind, 551, Hermann, Paris (1937).

[11] M.yaghoubi, Weil uniformity in fuzzy set theory, International Journal Of Pure and Applied Mathematics, 30, No. 4, 497-506. 
